\title{
Wearable Pressure Sensor Array for Health Monitoring
}

\author{
Matti Kaisti ${ }^{1}$, Joni Leppänen ${ }^{2}$, Olli Lahdenoja ${ }^{1}$, Pekka Kostiainen ${ }^{2}$, \\ Mikko Pänkäälä ${ }^{1}$, Ulf Meriheinä ${ }^{2}$, Tero Koivisto $^{1}$ \\ ${ }^{1}$ Department of Future Technologies, University of Turku, 20500 Turku, Finland \\ ${ }^{2}$ Murata Electronics OY, Vantaa, Finland
}

\begin{abstract}
Measuring the arterial waveform in real-time using wearable devices mounted directly on skin holds promise in assessing cardiovascular health status and detecting an early onset of cardiovascular disease. We report the use of modern high performance MEMS pressure sensors for wearable health monitoring. The low-cost sensor elements were incorporated onto a flexible wristband for radial artery pulse measurement. These sensor elements were configured as an array and attached to a wristband. The device operation was tested on 13 healthy subjects and from each subject we successfully derived the average arterial waveform, located the diastolic and systolic peaks together with Dicrotic notch and calculated the heart rate. In the future, the MEMS pressure sensors might be employed for mobile and remote cardiovascular health monitoring.
\end{abstract}

\section{Introduction}

The first arterial waveform measurements were conducted already in the late 19th century. A device called sphygmograph was used to measure blood pressure. This mechanical device was soon replaced with a cuff based method that is still prominently used today and is one of the most established diagnostic tools for blood pressure monitoring. However, it is designed to record only two pressure values, the systolic and diastolic pressure. It can not be used for continuous measurement although the latest commercial devices are able to detect irregular beats (e.g. atrial fibrillation) and morning hypertension. By recording the arterial waveform a wealth of information about cardiovascular health and aging can be extracted. The arterial waveform analysis allows the calculation of many parameters related to health. A modern technique for this purpose is called applanation tonometry where a sensor is pressed against an artery by a trained physician, and the pressure of the blood circulation is registered. These systems, however, are not designed for wearable nor continuous use.

A related techniques for blood pressure and wave- form sensing include the use infrared photoplethysmogram (PPG) placed e.g. in the finger with an inflatable cuff. The blood volume changes can then be recorded with the help of pneumatic valve [1] and controller electronics. Blood pressure can also be computed from the pulse transmit time (PTT) when several sensing points are utilized (see e.g. [2]). One alternative to PPG for health status monitoring is pressure sensing and the recent developments include the following; a pressure sensor elements have been created using polymer transistors [3], gold nanowires [4] and piezoelectric materials [5] for measuring blood pulse waveform. For health parameter extraction an automatic delineation of arterial blood pressure waveform was developed [6] and a single fiducial point method for long term blood pressure monitoring has been conceptualized [7].

We concentrate on the waveform analysis of a noninvasive wearable and continuous-time MEMS arterial pressure sensing device. The use of modern MEMS sensor technologies have high performance in a compact package with low power consumption. These sensors can be seamlessly integrated into modern electronic devices. Since the measurement procedure is sensitive to the exact placement of the sensor to an artery, it is preferable to have several sensors arranged into a grid and use an automated selection of the best MEMS sensor from the multiple of sensing elements. With each recording a high quality signal was obtained from the sensor array and subsequently the heart rate was computed and arterial waveform extracted indicating the sensors potential for wearable health monitoring in the future.

\section{Methods}

Sensor operation principle: Murata's capacitive pressure element (SCB10H) consists of two silicon wafers and one glass wafer. One silicon wafer is the sensors diaphragm that bends with external pressure. This pressure is proportional to the amount of bending and therefore the change in distance between two electrodes alters the sensors capacitance. The element is an absolute pressure sensor and the typical dynamic range is $4 \mathrm{pF}$ or $50 \%$ of the base capac- 


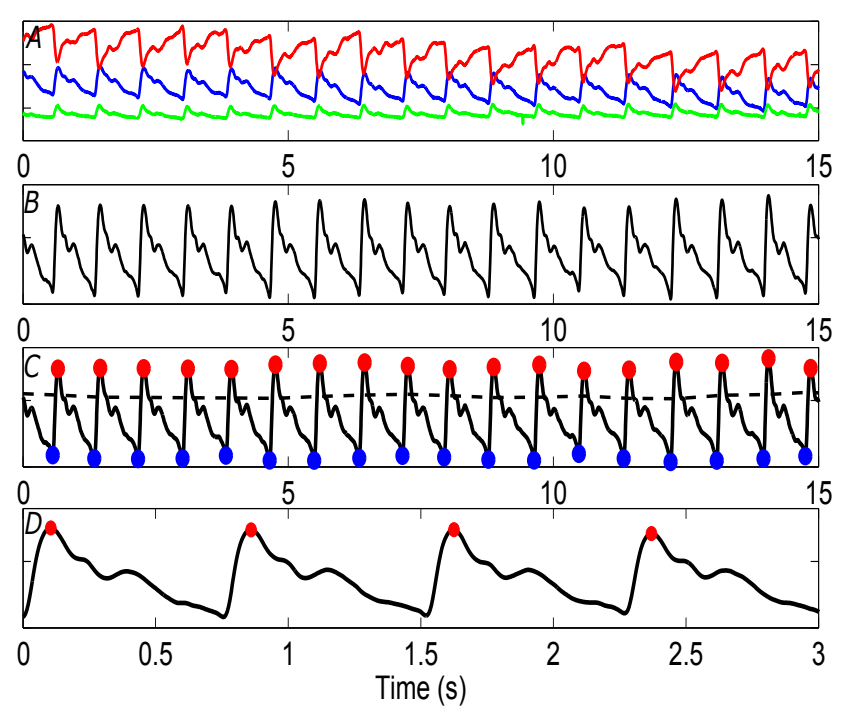

Figure 1: A) Raw signal traces from the array. B) The selected signal after band-pass filtering. C) Detected peaks (red), corresponding valleys (blue) and adaptive threshold (dashed line) which was used to determine whether each peak candidate is a true peak or a noise peak. D) A zoom of C) with only found peaks indicated (red).

itance. It is only limited by the allowed maximum nonlinearity associated with the hyperbolic relationship between electrode distance and capacitance value. This creates a significantly high dynamic range and gives a unique combination of high resolution and low current consumption. Murata's capacitive design in a single crystal silicon also results in minimized material stress, maximized overload performance and has practically no plastic deformation. When the element is pressed against an artery the obtained pressure value is comprised from counterforce of the hold down pressure as well as from the transmural pressure from the blood vessel [8]. The measured pressure is a sum of these components. The hold down pressure in an ideal case remains constant. Movement and arm tension can create significant changes in this pressure component causing difficulty in detecting the pulse waveform.

Signal acquisition system: The pressure sensor housing was covered with gel for coupling the blood pressure pulse signal to the sensor element. The change in the sensor capacitance was read with Silicon labs EFM32, a 32-bit ARM Cortex-M3 core based microcontroller, using the Acam PCap04Z capacitance-to-digital converter. A Python application was used to acquire the signal from the microcontroller to the PC via RS232 serial interface.

Data collection: Three pressure sensor elements (SCB10H Murata) were assembled on a flexible wristband. The band was wrapped around the left wrist and positioned roughly over the radial artery. The output was digitized and send to PC for post processing. Data was collected from 13 healthy subjects between 25 and 53 years of age. All subjects were in a seated position with the arm in a relaxed position. The sensor array and the wristband placement are shown in Figure 2 b).

\section{Algorithm}

Preprocessing and sensor selection: The preprocessing of each sensor element signal include decimating it from $685 \mathrm{~Hz}$ to $200 \mathrm{~Hz}$ signal, removing the mean values of the signal and clipping spurious glitches against predetermined threshold. From the preprocessed signal we compute all local peaks and valleys that have atleast $0.5 \mathrm{~s}$ separation. The median value is computed for the peaks and valleys from which a single peak-to-peak value is computed. This value is divided by the standard deviation of the high-pass $\left(f_{c}=50 \mathrm{~Hz}\right)$ filtered version of the signal. The signal with the highest value is considered to have the highest signal-to-noise ratio and is selected for further processing. The selected signal was band-pass filtered using a third order Butterworth zero-phase IIR filter with cut-off frequencies of $1 \mathrm{~Hz}$ and $20 \mathrm{~Hz}$. In addition to high frequency noise the filter removes bias and partly the baseline wandering.

Peak detection: After filtering the selected signal the peaks are detected using an automatic multiscale-based peak detection (AMPD) algorithm [9]. The algorithm constructs a matrix consisting of scale-dependent local maximas. The details for the peak detection are provided in [9]. The found local maximas are considered as peak candidates and a search-back routine is initiated that determines whether the candidate is either a noise or a true peak. This step is based on adaptive thresholding. For each peak candidate amplitude $P_{a}$ the threshold is determined by a weighted average of $P_{a}$ and current threshold $\left(T H_{i}\right)$ by $T H_{i+1}=1 / 3 \times P_{a}+2 / 3 \times T H_{i}$. Subsequently the $T H$ is updated if the following condition of a true peak is satis-

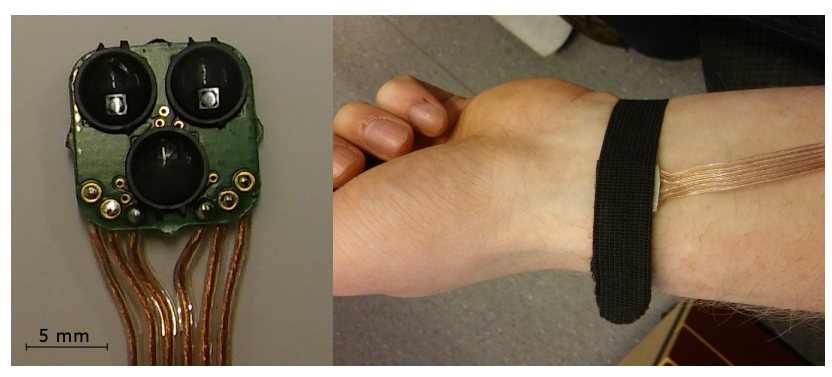

(a)

(b)

Figure 2: a) MEMS pressure sensor array configuration on small PCB with interface circuitry. b) The wearable wristband placement during measurement. 
fied, $P_{a}>0.7 \times T H_{i+1}$. We do not initiate a search-back if a peak is clearly missed as the purpose of the algorithm is to robustly find correct peaks for waveform analysis rather than accurate heart rate estimation although the algorithm finds peaks from the test data set with high performance.

Average waveform: The peaks were used as fiducial points for waveform ensemble averaging. The average time difference of preceding valleys before each peak was used to compute the window length for the waveform. Each obtained waveform (one for each found peak) were aligned by matching the peaks. Waveforms deviating more than two standard deviations from the mean were discarded. The remaining waveforms were ensemble averaged and the result is the final pulse waveform.

Waveform analysis: From the obtained waveform the signal was spline interpolated to $1000 \mathrm{~Hz}$ and normalized to have a maximum of one. The dicrotic notch was determined by fitting a first order polynomial with a least squares mean from the the diastolic peak onward and substracting this line from the original signal. This minimum point serves as another fiducial point that allows finding the dicrotic notch from the original waveform. This is achieved by computing the minima from a narrow window surrounding the fiducial point. Furthermore, the 1st and 2nd order derivatives are computed and the zero crossings and local maxima and minima are computed from them, respectively. The dicrotic notch, systolic and diastolic peaks can also be computed from the signal derivatives as indicated in Figure 3. Combining these methods allows more robust parameter estimation with inter-subject variability.

\section{Results and Discussion}

Sensor selection: The array configuration provides more robust sensing and easier sensor placement for obtaining pulse waveform from the wrist compared to single element configurations. This, however, adds the requirement to either combine or select the best element from the array. We developed an sensor selection method. In the 13 measurements the quality was evaluated via an expert opinion. The expert considers the following quality metrics: ability to detect peaks clearly, the shape of the pulses and the amount of high frequency noise. In $85 \%$ of the cases the algorithm and expert opinion match. In the remaining two cases the algorithm chooses an element that is considered only slightly sub-optimal. In these cases two elements provide a high quality signal. In all cases a clear signal waveform and heart rate can be obtained.

Heart-rate detection: The peak detection algorithm was evaluated by comparing the detected peaks to an expert annotation. The detection performance of heartbeats were evaluated by calculating the positive predictive value (PPV), and the true positive rate (TPR). The results are col-

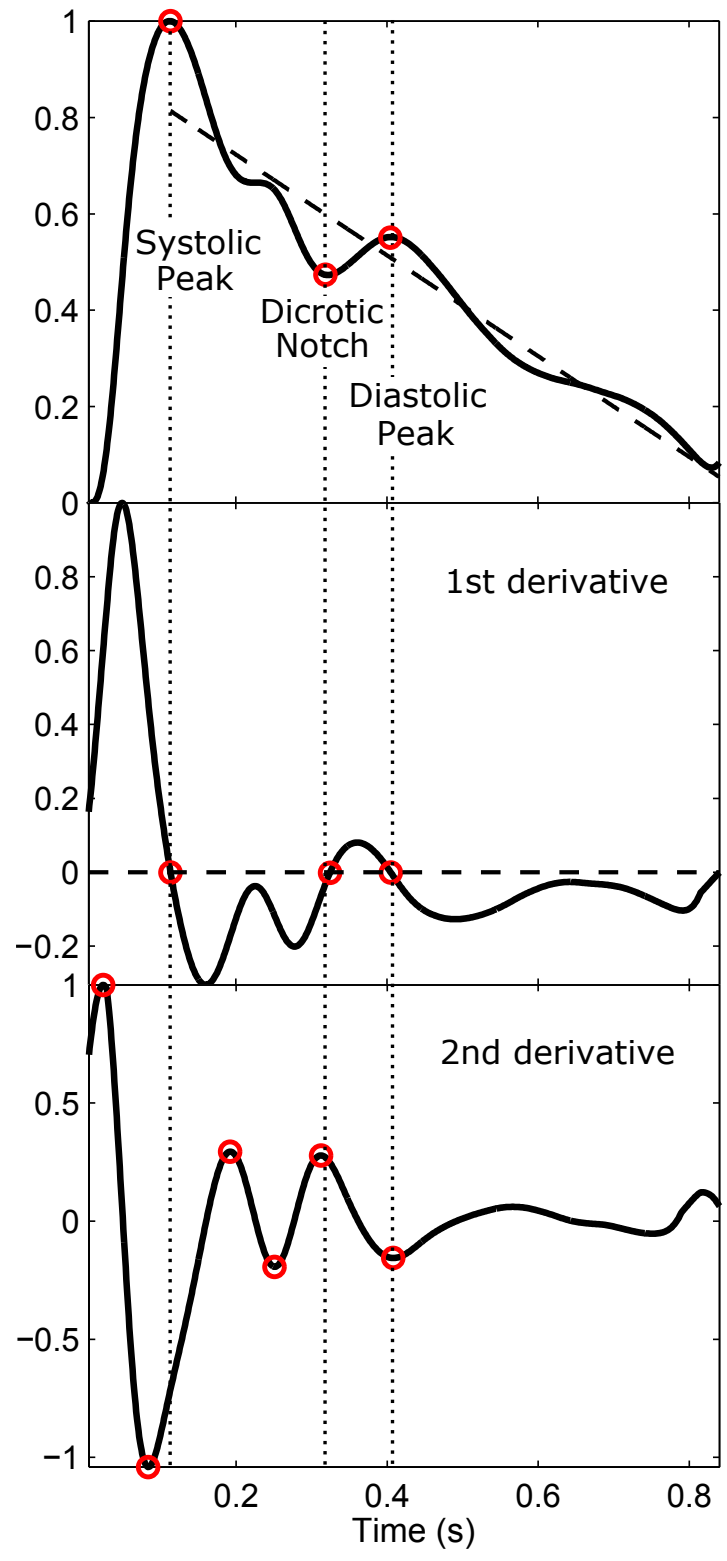

Figure 3: a) Extracted ensemble averaged waveform. b) The 1st derivative of the waveform. c) 2nd derivative of the waveform. Systolic and diastolic peaks and dicrotic notch are indicated in the figure with (red) circles.

lected to Table 1 where the high performance of the detection is evident. In most cases, the high signal quality simplifies the actual detection significantly. These rates are, however, expected to be clearly worse in a less controlled environment and in the presence of motion artefacts.

Radial artery pulse measurement: One of the sensor limitations arise from the hand movement and sensor positioning. Any tension in the arm affects the sensor output via changes of pressure by either conformal changes 
Table 1: Performance metrics of the beat-to-beat detection. HR, TPR and PPV stand for heart rate, true peak rate and positive predictive value respectively. Bottom line shows the mean of each metric.

\begin{tabular}{lccc} 
ID & HR (bpm) & TPR $(\%)$ & PPV $(\%)$ \\
\hline 1 & 90.3 & 96.9 & 99.5 \\
2 & 72.0 & 100 & 100 \\
3 & 67.5 & 100 & 100 \\
4 & 91.8 & 92.7 & 100 \\
5 & 57.5 & 99.2 & 100 \\
6 & 93.4 & 100 & 100 \\
7 & 61.6 & 100 & 100 \\
8 & 107.6 & 100 & 100 \\
9 & 96.9 & 100 & 100 \\
10 & 69.9 & 100 & 100 \\
11 & 60.8 & 100 & 100 \\
12 & 60.2 & 99.2 & 100 \\
13 & 74.3 & 100 & 100 \\
\hline $\bar{x}$ & 77.2 & 99.1 & 100 \\
\hline
\end{tabular}

under the sensing area of changes via the arm diameter causing strap to be pressed against the arm with different force. The operation in the current configuration is mainly limited to still operation. This, however, only partly limits the usability as the waveform can be obtained using a very short measurement time. An example figures for the ensemble averaged waveform and its 1 st and 2 nd order derivatives is presented in Figure 3. These allows annotating and deriving the determination of important waveform parameters. We demonstrate the extraction of the systolic and diastolic peaks and dicrotic notch. The relation of these parameters to cardiovascular health has been reviewed [10].

\section{Conclusion}

The presented wearable device and the accompanying algorithm indicate that MEMS pressure sensors could be used for future wearable skin-mounted sensors for longterm and unobtrusive monitoring. The sensor operation requires little or no training and can be fabricated with low-cost. The high performance operation of the MEMS devices provide side-effect free daily operation and the plastic cover ensures that there is no skin irritation. The downside of the sensing configuration is its sensitivity to hand movement and muscle tension which cause significant amplitude variations to the signal. Thus, the system is probably limited to applications where it suffices to take continuous point in time measurements. The applicability of the sensor array and accompanying algorithm was demonstrated via sensor element selection after which the heart beats could be robustly detected together with a clean waveform. Future work includes investigating the ability to extract artefacts from the signal, detection of atrial fibrillation and a comparison study with invasive pressure sensors.

\section{References}

[1] Parati G, Casadei R, Groppelli A, Di Rienzo M, Mancia G. Comparison of finger and intra-arterial blood pressure monitoring at rest and during laboratory testing. Hypertension 1989;13(6 Pt 1):647-655.

[2] Gesche H, Grosskurth D, Küchler G, Patzak A. Continuous blood pressure measurement by using the pulse transit time: comparison to a cuff-based method. European journal of applied physiology 2012;112(1):309-315.

[3] Schwartz G, Tee BCK, Mei J, Appleton AL, Kim DH, Wang $\mathrm{H}, \mathrm{Bao} \mathrm{Z}$. Flexible polymer transistors with high pressure sensitivity for application in electronic skin and health monitoring. Nature Communications 2013;

[4] Gong S, Schwalb W, Wang Y, Chen Y, Tang Y, Si J, Shirinzadeh B, Cheng W. A wearable and highly sensitive pressure sensor with ultrathin gold nanowires. Nature Communications 2014;

[5] Dagdeviren C, Su Y, Joe P, Yona R, Liu Y, Kim YS, Huang Y, Damadoran AR, Xia J, Martin LW, Huang Y, Rogers JA. Conformable amplified lead zirconate titanate sensors with enhanced piezoelectric response for cutaneous pressure monitoring. Nature Communications 2014;

[6] Li BN, Dong MC, Vai MI. On an automatic delineator for arterial blood pressure waveforms. Biomedical Signal Processing and Control 2010;5(1):76-81.

[7] Addison PS. Slope transit time (stt): A pulse transit time proxy requiring only a single signal fiducial point. IEEE Transactions on Biomedical Engineering Nov 2016; 63(11):2441-2444.

[8] Zuo W, Wang P, Zhang D. Comparison of three different types of wrist pulse signals by their physical meanings and diagnosis performance. IEEE Journal of Biomedical and Health Informatics Jan 2016;20(1):119-127. ISSN 21682194.

[9] Scholkmann F, Boss J, Wolf M. An efficient algorithm for automatic peak detection in noisy periodic and quasiperiodic signals. Algorithms 2012;5(4):588-603.

[10] Elgendi M. On the analysis of fingertip photoplethysmogram signals. Current Cardiology Reviews 2012;.

Address for correspondence:

Matti Kaisti

Department of Future Technologies, University of Turku

Vesilinnantie 5, 20500 Turku, Finland

mkaist@utu.fi 